\title{
De la ecología política a una geografía social y política del ambiente
}

\author{
Julien Rebotier
}

\section{(2) OpenEdition \\ 1 Journals}

Edición electrónica

URL: http://journals.openedition.org/bifea/924

DOI: $10.4000 /$ bifea.924

ISSN: 2076-5827

Editor

Institut Français d'Études Andines

Edición impresa

Fecha de publicación: 1 abril 2013

Paginación: 121-126

ISSN: 0303-7495

\section{Referencia electrónica}

Julien Rebotier, « De la ecología política a una geografía social y política del ambiente », Bulletin de I'Institut français d'études andines [En línea], 42 (1) | 2013, Publicado el 01 agosto 2013, consultado el 06 noviembre 2020. URL : http://journals.openedition.org/bifea/924 ; DOI : https://doi.org/10.4000/ bifea.924

\section{(c) $(7)(9$}

Les contenus du Bulletin de l'Institut français d'études andines sont mis à disposition selon les termes de la licence Creative Commons Attribution - Pas d'Utilisation Commerciale - Pas de Modification 4.0 International. 


\title{
De la ecología política a una geografía social y política del ambiente
}

\author{
Julien Rebotier*
}

\section{POSTURA Y TEMAS DE INVESTIGACIÓN}

Se puede concebir la ecología política como una plataforma de investigación cuyas problemáticas centrales giran en torno al medio ambiente. Los diferentes abordajes académicos que abarca son sumamente diferentes, y es de notar que algunos se plantean como incompatibles. Hay quienes ponen de realce las dinámicas ambientales y la necesidad imperiosa de entenderlas y manejarlas con inteligencia y perspicacia (Burton et al., 1978), y hay quienes hacen hincapié en la dimensión social y política del medio ambiente (o sea que el ambiente solo cobra interés en función del sentido y de las implicaciones que representa a través de y para la sociedad [Peet \& Watts, 2004]). El abanico de los abordajes se distribuye entre aquellos dos puntos de partida distintos de la reflexión, que no necesariamente son excluyentes, pero que sí nutren debates intensos (Vayda \& Bradley, 1999).

Mi práctica de geógrafo de los riesgos y del ambiente me hace adoptar una perspectiva sumamente social y política. Es decir que me aproximo a, e investigo problemáticas ambientales a partir de un territorio (entendido como un conjunto de contextos sociales, históricos y espaciales). El punto de partida, tanto de mis problemáticas como de mis investigaciones, radica en la organización y el funcionamiento de la sociedad (O'Keefe et al., 1976; Ribot, 2010). Tanto unas como otras son situadas, historicizadas y localizadas (Rebotier, 2011a). Los riesgos

* Geógrafo CNRS-SET (UMR 5603), Université de Pau et des Pays de l'Adour Investigador Asociado al IFEA. E-mail: julien.rebotier@univ-pau.fr 
ambientales y la adaptación al cambio climático son los temas mayores de mi trabajo. No parto de la amenaza ni del diagnóstico físico del cambio climático, sino que reflexiono a partir de lo que todo ello representa para la sociedad, la manera cómo se identifica el «problema» ambiental, cómo se mide y cómo se gestiona, y lo que implica en términos de relaciones sociales y de gestión del espacio. Socializar y politizar la geografía del ambiente no significa eludir las dinámicas biofísicas (Rebotier, 2012a). A pesar de no partir de ellas, constituyen variables al igual que muchas otras (marcos conceptuales dominantes, régimen de dominación o igualdad, acceso a los recursos o a la decisión, distribución diferenciada de impactos o riqueza), a veces son imperiosas (no se elude la ley de la gravedad, por ejemplo), pero nunca formatean las preguntas formuladas ni limitan el espacio estudiado (no busco entender mejor la mecánica de la amenaza o el balance biofísico del cambio climático en tal sector, ni trabajo dentro de los límites de una «cuenca peligrosa» o de un área potencial de extensión de un alud). La producción del ambiente constituye el enfoque teórico privilegiado (Rebotier, 2012b). No solo se busca saber cómo ocurren las cosas, sino por qué, siguiendo qué tipo de mecanismos o qué lógicas, asumiendo la hipótesis según la cual aquellos procesos no son ni totalmente contingentes, ni totalmente aleatorios (Smith \& O'Keefe, 1980). Dicha «producción» se entiende como una tensión entre la experiencia sensible y las capacidades de los individuos por un lado, y los marcos estructurantes, ofreciendo limitantes y oportunidades, por otro lado. El «ambiente» que me interesa es fruto de esta producción, función de una organización, una dinámica y un orden social, producto tanto de factores materiales como simbólicos (Godelier, 1984). La geografía del ambiente que practico ubica la explicación dentro de la sociedad y ambiciona contribuir al debate sobre justicia, democracia y responsabilidades (individuales y colectivas) frente a los desafíos ambientales, así como sobre las modalidades de gestión ambiental. De hecho, al gestionar el «medio» se gestiona tanto la sociedad como el ambiente (retomando la denominación moderna), siguiendo lógicas y jerarquías eminentemente situadas que me interesan investigar. La perspectiva de la producción del ambiente también es deliberadamente normativa y crítica, en la medida en que, a mi modo de ver, existen opciones o configuraciones mejores que otras, e.g. no todos los discursos se valen. Pero ello no implica que existan situaciones ni procesos idóneos, e.g. la conservación o la adaptación, de por sí, tienen poco significado si no se sabe para quién, por qué y en qué circunstancias. En esta dimensión constantemente dinámica de la producción del ambiente radica un interés fundamental para el análisis; pero también en términos políticos, de democracia, de gestión y de manejo ambiental.

De ahí, formulo algunas preguntas generales de investigación:

- ¿Cuáles son las condiciones que hacen a la gente vulnerable?

- ¿Cuáles son las implicaciones del uso y de la aplicación de categorías de análisis y de acción (como lo son vulnerabilidad, adaptación o resiliencia)?

- ¿Por qué «saber más» no necesariamente se traduce por «actuar mejor»? 
- ¿Cuál es el perímetro de las preguntas y potenciales soluciones que se ofrecen a una sociedad en el ámbito de los desafíos ambientales?

\section{LA INVESTIGACIÓN DEL MOMENTO: POLÍTICAS AMBIENTALES}

Tras una serie de trabajos de campo y publicaciones sobre los riesgos urbanos (más enfocados en la dimensión socio-ambiental [Rebotier, 2009] y gobernanza urbana [Rebotier \& Rivière d'Arc, en curso]) o la inseguridad urbana en Caracas (más enfocados en la dimensión política [Rebotier, 2011b]), el programa de investigación que más me ocupa actualmente consiste en estudiar los cambios ambientales y las estrategias de adaptación en la región Aquitaine - Francia.

El programa CESAR investiga dimensiones cruzadas de la adaptación al cambio climático en los territorios locales de Aquitaine. Por dimensiones cruzadas, se entiende la manera cómo la noción de adaptación circula a escala global y se ve localmente mediante políticas públicas (descendiente), y a partir de los habitantes, los profesionales, sus prácticas y representaciones, la manera cómo se concreta (o no) la idea de adaptación y la cuestión del cambio climático (ascendiente). De forma más epistemológica, el programa cuestiona el uso y la referencia a la adaptación al cambio climático, sus implicaciones para las sociedades y sus territorios, el encuentro o desencuentro entre aquellas dimensiones por un lado, y sus relaciones con la —antigua - idea de «cambio social» por otro lado, o cómo afianzar manejo del ambiente y transformaciones sociales (Pelling, 2011; Ribot, 2011).

Al final del primer año de trabajo (2012), y en base a observaciones de campo, entrevistas con técnicos territoriales y análisis de un corpus de documentos puestos en línea por los gobiernos locales de Aquitaine, podemos destacar tres puntos en relación con la dimensión descendiente de la adaptación (Rebotier, 2012c).

Primero, es necesario subrayar el peso normativo del marco legal y reglamentario. Los dispositivos se repiten de forma muy similar en las diferentes colectividades. Se notan los mismos planos, «balances-carbono» y documentos o folletos, prueba de la aplicación indiferenciada de «estrategias» (o al menos «recetas») de adaptación. Segundo, se hace mucho hincapié en la necesidad de transversalidad de la adaptación como filtro previo imprescindible para pensar los demás sectores de gestión pública (el mainstreaming). Al revés, en muy pocos casos se reconoce la pluralidad de los desafíos que conlleva la adaptación, y que a menudo quedan escondidos por el carácter poco cuestionable de la noción (por ejemplo: redistribución de poderes en un proceso continuo de descentralización, capacidad de priorizar una agenda política, apertura de nuevos mercados o capacidades de adaptación diferenciadas). En base a nuestro enfoque de producción del ambiente, aquellos desafíos no son periféricos a, sino constitutivos de la cuestión socio-ambiental, en este caso de la adaptación.

Tercero, al ser la adaptación una categoría de análisis y de acción a la vez, la posible disimulación de desafíos sociales y políticos cobra aún más importancia. Se nota la dimensión preformativa del formateo de las preguntas y potenciales 
soluciones evocadas en relación con la adaptación ya que se concretan en el espacio y para la gente a través de políticas y dispositivos (disposiciones fiscales, reglamentos, carga simbólica o estigmatizadora).

En el campo es obvio como ya no se puede concebir los poderes públicos de forma descendiente, como productores exclusivos de norma y legitimidad. Nuevos actores emergen (ONG, asociaciones, habitantes) y cuestionan los dispositivos y las prioridades de gestión ambiental. Es fundamental «situar» (en el espacio, en un momento histórico, y en un grupo social particular) la adaptación en los diferentes territorios de Aquitaine. La problemática del cambio climático y las políticas relacionadas no solo varían en función del clima. También la constituyen y resultan afectadas aspectos políticos, sociales y culturales que son objetos del programa CESAR.

Los próximos pasos son de doble filo. Unas consideraciones epistemológicas llevan a plantear de manera social y política la adaptación, y consolidar un enfoque epistemológico-metodológico de producción del ambiente. Unas consideraciones políticas y prácticas orientan la investigación hacia la elaboración de dispositivos de gestión territorial que permitan concretar iniciativas de adaptación situadas.

\section{APERTURA Y PERSPECTIVAS}

Están emergiendo colaboraciones con Brasil, Quebec y Venezuela. En Brasil, con colegas de la Unicamp, se busca conocer mejor, y de manera comparativa, los marcos conceptuales que fundamentan las políticas ambientales desarrolladas en el ámbito del cambio climático. También se intenta caracterizar las modalidades, limitantes y oportunidades de aplicación de dichas políticas en los terrenos de Aquitaine (Francia) y Campinas y Limeira (estado de São Paulo, Brasil). En Quebec, con una colega involucrada en desarrollo regional, en la UQAR, se inicia un diálogo metodológico y comparativo. Son diferentes proyectos de ordenamiento ambiental y territorial en dos contextos regionales del «norte» (Bajo Saint Laurent, Quebec y Aquitaine, France). Buscamos articular metodologías más estructurantes con otras más enfocadas en los individuos, en la capacidad de actuar de los grupos y en las configuraciones locales. Se ambiciona así consolidar metodológicamente el enfoque de investigación mediante la perspectiva de la producción del ambiente. En Venezuela, se inicia una colaboración temática que consiste en evaluar la actividad agropecuaria de montaña (entre Andes venezolanos y Pirineos occidentales franceses) en el contexto del cambio climático. Aprovechando la idea de la producción del ambiente como una plataforma posible de diferentes planteamientos, los colegas involucrados en la investigación contribuyen a documentar los aspectos de geociencia, de geografía social y agraria, humanista y cultural, o social y política de la actividad agropecuaria y de la manera cómo está directa o indirectamente afectada tanto por el cambio climático como por las políticas y discursos relacionados. El interrogante principal que nutre aquellas colaboraciones consiste en entender cómo y por qué en diferentes territorios, diferentemente insertos en la globalización, se actúa frente a una problemática 
ambiental global (el cambio climático), que se traduce localmente de manera diferenciada.

Más allá de las investigaciones específicas y cooperaciones iniciadas, una hipótesis más integradora consiste en afirmar que, a pesar de la dimensión global de muchas de las problemáticas ambientales, las diferencias no solo dependen de las diferentes configuraciones o dinámicas ambientales a escala local, sino que también corresponden a las características de la organización y del funcionamiento social. De ser así, existen ramificaciones ulteriores hacia 1) la caracterización de un desarrollo y un manejo ambiental diferenciado (a través de la manera cómo los territorios se articulan a diferentes escalas a la problemática ambiental global); así como 2) la contribución al debate sobre la recomposición de la modernidad y la práctica de las ciencias sociales en el contexto de una problemática ambiental, tanto federativa (afirmación y experiencia de un devenir común, de lo que es y significa la Humanidad) como discriminatoria (quiénes ganan y quiénes pierden, cuáles son los elementos incluidos en la idea de justicia y a qué escala, en relación con los desafíos ambientales contemporáneos).

\section{Referencias citadas}

BURTON, I., KATES, R. \& WHITE, G., 1978 - The Environment as Hazard, 290 pp.; NewYork: Oxford University Press.

GODELIER, M., 1984 - L'idéel et le matériel, 300 pp.; París: Fayard.

O'KEEFE, P., WESTGATE, K. \& WISNER, B., 1976 - Taking the naturalness out of natural disasters. Nature, 260 (5552): 566-567.

PEET, R. \& WATTS, M., (eds.) 2004 - Liberation ecologies. Environment, development, social movements, 288 pp.; London and New York: Routledge.

PELLING, M., 2011-Adaptation to climatechange. From resilience to transformation, 224 pp.; London \& New York: Routledge.

REBOTIER, J., 2009 - La dimensión territorial de los riesgos urbanos en Caracas. La pertinencia de las ciencias sociales. Ecología política y construcción social. In: EIRD Informa: http://www.eird.org/plataforma-tematica-riesgo-urbano/recopilacion-de articulos/index.html

REBOTIER, J., 2011a - Habiter les territoires et construire les risques, entre empreintes spatiales et logiques sociales - Caracas. In: Habiter les territoires à risque (V. November, M. Penelas \& P. Viot, eds.): 81-100; Lausanne: Éditions Presses Polytechniques et Universitaires Romandes.

REBOTIER, J., $2011 \mathrm{~b}$ - Politicizing fear of crime and insecurity in Caracas. The manufacturing of a fearful urban meta-narrative. Emotion, Space and Society, 4 (2): 104-112.

REBOTIER, J., 2012a - Vulnerability conditions and risk representations in LatinAmerica: Framing the territorializing urban risk. Global Environmental Change, 22 (2): 391-398. 
REBOTIER, J., 2012b - La production de l'environnement. Colloque « Géographie, écologie, politique : un climat de changement ». Orléans, 6-8 septembre.

REBOTIER, J., 2012c - Quelles questions l'adaptation au changement climatique poset-elle sur les territoires aquitains ? Journée d'étude "Adaptation au changement environnemental en Aquitaine. Politiques publiques et pratiques locales : quelles rencontres? ». Pau, 14 décembre.

REBOTIER, J. \& RIVIÈRE D'ARC, H., en curso - Transformations sociopolitiques et gouvernance métropolitaine dans le domaine du logement en Amérique latine. Comprendre et donner du sens à la gouvernance. In: Modes de gouvernance dans quatre métropoles latino-américaines (Buenos Aires, Caracas, Mexico et São Paulo) : entre logiques institutionnelles et acteurs (C. Azaïs, C. Bidou, J. Devineau, M. Pépin Lehalleur, J. Rebotier \& H. Rivière d'Arc, eds.); Peter Lang.

RIBOT, J., 2011 - Vulnerability before adaptation: toward transformative climate action. Global Environmental Change, 21 (4): 1160-1162.

RIBOT, J., 2010 - Vulnerability does not fall from the sky. Towards a multiscale, pro-poor climate policy. In: Social Dimensions of climate change (R. Mearns \& A. Norton, eds.): 47-74; Washington: The World Bank.

SMITH, N. \& O'KEEFE, P., 1980 - Geography, Marx and the concept of nature. Antipode, 12(2): 30-39.

VAYDA, A. P. \& BRADLEY, B. W., 1999 - Against Political Ecology. Human Ecology, 27 (1): 167-179. 